\title{
Self-aligned and bundled electrospun fibers prepared from blends of polystyrene (PS) and poly(methyl methacrylate) (PMMA) with a hairy-rod polyphenylene copolymer
}

\author{
Tamer Uyar ${ }^{\text {a,e,* }}$, Ioan Cianga ${ }^{\text {b,c }}$, Luminita Cianga ${ }^{\text {b,c }}$, Flemming Besenbacher ${ }^{\text {a,d }}$, Yusuf Yagci ${ }^{\text {b,* }}$ \\ a Interdisciplinary Nanoscience Center (iNANO), Aarhus University, DK-8000, Aarhus C, Denmark \\ ${ }^{b}$ Department of Chemistry, Istanbul Technical University, Maslak, TR-34469, Istanbul, Turkey \\ c "Petru Poni" Institute of Macromolecular Chemistry, Iasi 700487, Romania \\ d Department of Physics and Astronomy, Aarhus University, DK-8000, Aarhus C, Denmark \\ e UNAM-Institute of Materials Science and Nanotechnology, Bilkent University, 06800, Ankara, Turkey
}

\section{A R T I C L E I N F O}

\section{Article history:}

Received 5 March 2009

Accepted 28 April 2009

Available online 3 May 2009

\section{Keywords:}

Electrospinning

Hairy-rod conjugated copolymer

Aligned nanofiber

Polyphenylene

Polystyrene (PS)

Poly(methyl methacrylate) (PMMA)

\begin{abstract}
A B S T R A C T
Bundled and self-aligned fibers were obtained by electrospinning blends of polystyrene (PS) and poly (methyl methacrylate) (PMMA) with a hairy-rod polyphenylene-g-polystyrene/poly(a-caprolactone) (PP-gPS/PCL) copolymer. The self-alignment and bundling characteristics of these electrospun fibers were ascribed to the unique molecular architecture of the conjugated polymer, PP- $g$-PS/PCL, and its interactions with the solvent and the polymer matrixes used for the electrospinning. The self-alignment and bundling was found to be much more pronounced for PP-g-PS/PCL-PS blend when compared to PP-g-PS/PCL-PMMA blend. Furthermore we found that the degree of self-alignment of the fiber bundles was enhanced by increasing the amount of PP- $g$-PS/PCL in the blends but the alignment completely disappeared when the solvent dimethylformamide was changed to chloroform.
\end{abstract}

(c) 2009 Elsevier B.V. All rights reserved.

\section{Introduction}

Electrospinning is a very versatile and cost effective technique for producing multi-functional nanofibers from various polymers, polymer blends and composite solutions, etc. [1-3]. Electrospun fibers of conjugated polymers have received attention due to their unique properties and potential applications as electrical and optical materials [4-8]. However, most of the cases the intractability of conjugated polymers prohibit the electrospinning of fibers by themselves, so fibers of conjugated polymers have mainly been obtained by blending with other polymers which can be easily electrospun [4,5].

In this study we attempted to electrospin fibers from hairy-rod conjugated copolymer, polyphenylene-g-polystyrene/poly(a-caprolactone) (PP-g-PS/PCL), however, the electrospinning of pure PP- $g-\mathrm{PS} / \mathrm{PCL}$ yielded only nano or micron size particles instead of fibers. Nonetheless fibers were successfully electrospun from PP-g-PS/PCL when blended with polystyrene (PS) or poly(methyl methacrylate) (PMMA). Surprisingly, it was observed that the electrospun PP-g-PS/PCL-PS and PP-g-PS/ PCL-PMMA fibers were bundled and self-aligned when collected on a stationary flat sheet collector. Based on the experimental findings the self-alignment and the bundling characteristics of the PP-g-PS/PCL-PS

\footnotetext{
* Corresponding authors. Uyar is to be contacted at Tel.: +90 312 2903571; fax: +90 312 2664365. Yagci, Tel.: +90 212285 3241; fax: +90 2122856386.

E-mail addresses: tameruyar@unam.bilkent.edu.tr (T. Uyar), yusuf@itu.edu.tr (Y. Yagci)
}

and PP-g-PS/PCL-PMMA fibers were attributed to the unique molecular architecture of the conjugated polymer, PP-g-PS/PCL, and its interactions with the solvent ( $N, N$-dimethylformamide (DMF)) and the polymer matrixes (PS and PMMA). Additionally, to the best of our knowledge, this is the first report on electrospinning of fibers derived from polyphenylenes (PPs) or their derivatives.

\section{Experimental}

\subsection{Materials}

Polystyrene (PS) $\left(\mathrm{M}_{\mathrm{w}} \sim 280,000\right.$, Sigma), poly(methyl methacrylate) (PMMA) $\left(\mathrm{M}_{\mathrm{w}} \sim 350,000\right.$, Aldrich) and $N, N$-dimethylformamide (DMF) (Fluka, 98\%) were used as purchased. The poly-p-phenylene 3 (PP-g-PS/PCL) (Scheme 1) containing both PS and PCL side chains, was synthesized by Yamamoto co-polycondensation. The synthesis details together with structural characterizations of PP-g-PS/PCL were previously reported $[9,10]$.

\subsection{Electrospinning}

The homogeneous solution of PMMA, PS, PP-g-PS/PCL, and the blends of PP-g-PS/PCL-PS and PP-g-PS/PCL-PMMA were prepared in DMF and then electrospun. The homogeneous polymer solutions were placed in a $1 \mathrm{ml}$ syringe fitted with a metallic needle $(0.4 \mathrm{~mm}$ inner diameter). The syringe was fixed horizontally on the syringe pump 


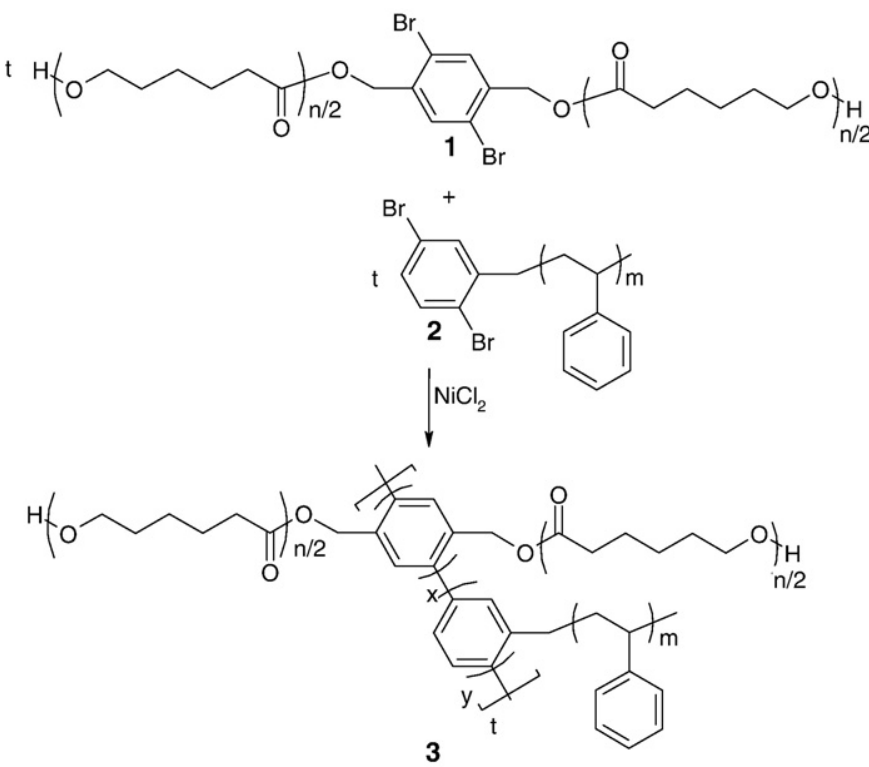

Scheme 1. Synthesis of polyphenylene-g-polystyrene/poly(e-caprolactone) copolymer (PP-g-PS/PCL) by Yamamoto polycondensation; $n=24.12, m=18.47 ; x=0.58 ; y=0.42$ $[9,10]$.

(Model: KDS 101, KD Scientific) and the high voltage power supply (Spellman, MP Series) was used. The applied electrospinning parameters were as follows; applied voltage $=15 \mathrm{kV}$, feed rate $=1 \mathrm{~mL} / \mathrm{h}$, and tip-tocollector distance $=10 \mathrm{~cm}$. A grounded stationary rectangular metal collector plate $(15 \times 20 \mathrm{~cm})$ covered by a piece of aluminum foil was used for the fiber deposition. The solution content and the morphologies of the resulting electrospun fibers are presented in Table 1 . In the case of PP$g$-PS/PCL-PS blend (1:1 w/w ratio), the solution was also prepared in chloroform for comparison and electrospun under the same conditions. The morphologies of the fibers were investigated by high resolution scanning electron microscopy (SEM) (FEI, Nova 600 NanoSEM).

\section{Results and discussion}

Table 1 shows the composition and the concentration of the polymer solutions which only yielded bead-free fibers. The resulting average fiber diameter (AFD) and the morphologies of the electrospun fibers are also given. The SEM images in Fig. 1 shows the electrospinning result for the pure polymer matrixes; PP-g-PS/PCL, PS and PMMA. Initially, the pure PP-g-PS/PCL was electrospun, however, the electrospinning of pure PP-g-PS/PCL has resulted in only nano and micron size particles instead of fibers and this result is most likely due to the low molecular weight characteristics of PP-g-PS/PCL (Fig. 1a). Pure PS and PMMA were electrospun easily and resulted in randomly oriented fibers in the form of non-woven (Fig. 1b-c). So, PP-g-PS/PCL was subsequently blended with PMMA and PS polymers that can be easily electrospun into fibers.

However, we found that for the same electrospinning conditions, the blends of PP-g-PS/PCL-PS (Fig. 2a-b) and PP-g-PS/PCL-PMMA (Fig. 2d-f) yielded fibers which were mostly bundled with some alignment. The self-alignment was found to be much more pronounced for PP-g-PS/PCL-PS blends when compared with PP-gPS/PCL-PMMA blends, and furthermore the self-alignment feature was enhanced by the increasing amount of PP-g-PS/PCL in the blends. To have a better understanding, the electrospinning of PP-g-PS/PCL-PS polymer blend was also performed in chloroform, and it was found that the morphology of the webs strongly depends on the solvent used. In the case of the chloroform the fibers were randomly oriented (Fig. 2c) whereas self-aligned and bundled fibers of PP-g-PS/PCL-PS were obtained when DMF was used as solvent (Fig. 2a-b).

The observed spontaneous bundling and alignment of the fibers at the macroscopic level may be a consequence of several factors acting simultaneously. One of them is related to the internal structuring by hierarchical self-assembly through microphase separation in a spatial cylindrical confinement [6] created by the polymers used as matrixes. The formation of such a cylindrical confinement may occur due to the specific interactions of the polymer matrixes (PS and PMMA) with the amphiphile PP-g-PS/PCL copolymer and with the solvent during the solvent evaporation and the final drying steps. In fact we can refer to a morphological transition during the electrospinning process from one type of the order in the solution to another type of the order in the fiber form. Although more detailed studies are needed, the formation of hierarchical self-assembly of the blends' structure in solution and in the fiber form at the molecular level is quite likely to occur. If we assume that in both the DMF solution and in the solid fiber state we have a cylindrical morphology of HR, namely the cylindrical micelles, this transition develops during solvent evaporation.

In our investigated blends, the amphiphile PP-g-PS/PCL has low molecular weight providing sufficient mobility for coalescence processes to occur prior to solidification of the fibers. Also it was shown that in the case of film morphology studied for the same conjugated PP-g-PS/PCL, the PS side chains can be found in the top layer [10] due to its lower surface energy when compared to PCL. In the electrospinning process the dynamics of a polymer blend is driven by the surface tension, since a reduction of the surface free energy is associated with the evaporative process. Indeed, we suppose that the completely dried form of the PS matrix is present on the surface of the fiber due to their lower surface energy while the immiscible $\mathrm{OH}$ ended PCL oligomeric side chains are oriented into the core of the aggregates. Strong intermolecular hydrogen bonds between $\mathrm{OH}$ groups are expected to stabilize the supposed supramolecular selfassembly. Moreover, the stretching of fibers in the electrospinning process may orient conjugated polymers molecules along the longitudinal direction of the fibers, thus increasing its charge-carrier mobility [5] and concomitantly facilitates the formation of continuous electrical conduction paths throughout the electrospun fiber segments. This will result in discharging the surface of the electrospun fibers and therefore, reducing the repulsion between the individual fibers during electrospinning process.

After the fiber has been ejected from the tip of the needle, two types of electrostatic forces mainly control its subsequent motion towards the collector plate: (i) the force exerted by the strong external field, and (ii) the force exerted by any adjacent charged fiber [2]. Generally, when a continuous stationary conductive plate is used as collector these

Table 1

The solution compositions and the diameter/morphology of the resulting electrospun fibers.

\begin{tabular}{|c|c|c|c|c|c|}
\hline Solutions & \% PMMA (w/v) & $\%$ PS (w/v) & $\%$ PP-g-PS/PCL (w/v) & Average fiber diameter $(\mu \mathrm{m})$ & Morphology \\
\hline$\overline{\mathrm{PP}-g-\mathrm{PS} / \mathrm{PCL}}$ & - & - & 50 & - & Nano and micron size particles \\
\hline PS & - & 25 & - & $1.959 \pm 0.162$ & Randomly oriented microfibers \\
\hline PP-g-PS/PCL-PS (0.5:1) & - & 15 & 7.5 & $0.803 \pm 0.138$ & Bundled nanofibers with some alignment \\
\hline PP-g-PS/PCL-PS ( $1: 1)$ & - & 15 & 15 & $1.051 \pm 0.160$ & Bundled nanofibers/microfibers with major alignment \\
\hline PMMA & 15 & - & - & $0.977 \pm 0.088$ & Randomly oriented nanofibers \\
\hline PP-g-PS/PCL-PMMA (0.5:1) & 10 & - & 5 & $0.441 \pm 0.134$ & Some bundled nanofibers \\
\hline PP-g-PS/PCL-PMMA (1:1) & 10 & - & 10 & $0.557 \pm 0.057$ & Bundled nanofibers with some alignment \\
\hline PP-g-PS/PCL-PMMA (2:1) & 10 & - & 20 & $1.495 \pm 0.137$ & Bundled microfibers with some alignment \\
\hline
\end{tabular}



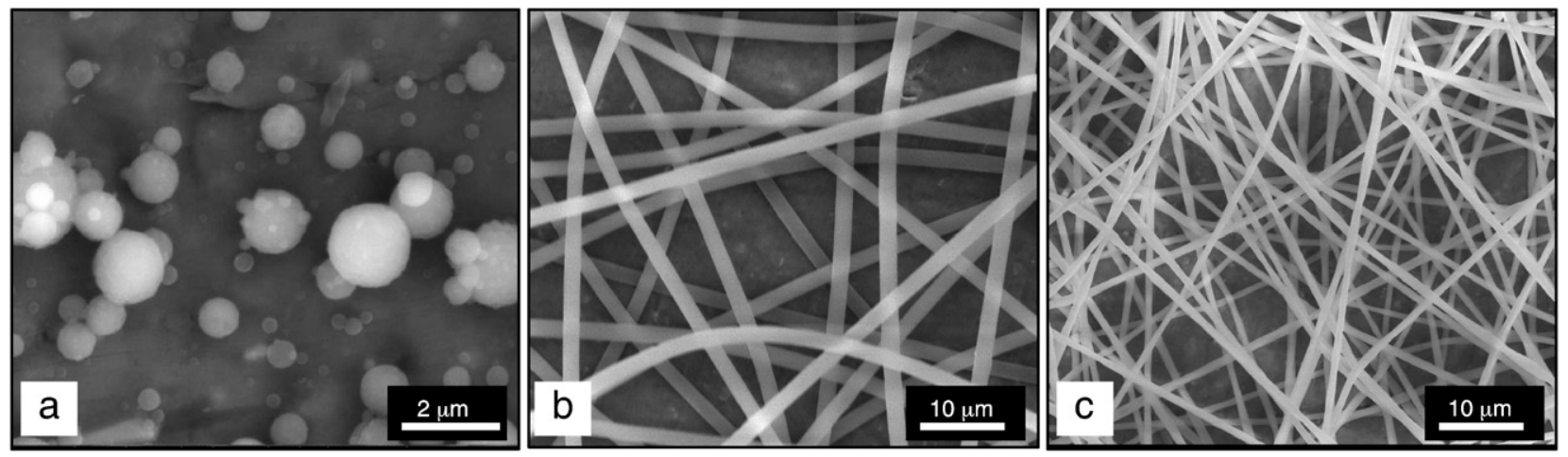

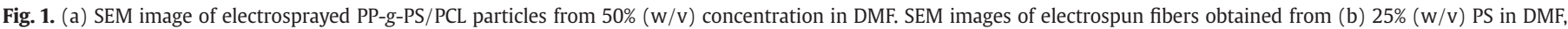
(c) $15 \%(w / v)$ PMMA in DMF.

electrostatic forces will have no preferential direction in the plane of the collector. So, in principle there should be no ordering/alignment for the collected fibers which were also observed for PS and PMMA used in this study (Fig. 1b-c). However, for the fibers produced from the PP-g-PS/ PCL-PS blends, uniaxially aligned bundles were observed spontaneously, and in this case the properties of the fibers may influence the abovementioned forces, most likely the electrical conductivity through the fibers since the charges can be adjusted by conduction [11]. The discharging of the surface of the electrospun fibers reduces the repulsion between the individual fibers during electrospinning process and therefore, fiber bundles can be formed. When the positive charge carrying fiber jet moves toward the negative collector, the bundle deposition pattern is expected to be directed by the intensity of its charge and by the repulsive forces already exerted on the deposited bundles. These collected charged fibers may repel the incoming similarly charged fibers and drive them towards the nearby conducting points on the collector for easier charge dissipation which preponderantly takes place through the electro-conducting conjugated self-assembled chains into the fibers of the bundles. The electrostatic repulsion between the deposited and incoming bundles may further enhance the parallel alignment since this morphology represents the lowest energy configuration for an array system of charged fibers [12]. Thus, the bundle formation and the self-alignment of the electrospun fibers from PP-g-PS/ PCL-PS blends may be explained as follows: (i) The highly charged viscous jets tightly looped and were preserved in the shape of solid fibers [13], (ii) The significant amount of the residual solvent can produce merged fibers upon reaching the collector [14]; this is due to the fact that the boiling point of DMF is fairly high, and there was not enough time for the solvent in the charged jet to completely evaporate before the "dried" fibers are deposited on the collector [15], (iii) The formation of the bundle and the self-alignment is due to the PS cylindrical confinement and the fact that it is probably located on the surface of the fibers.

As observed from the SEM images (Fig. 2d-f), the degree of bundling and alignment is low when PMMA is used as a matrix, as compared to
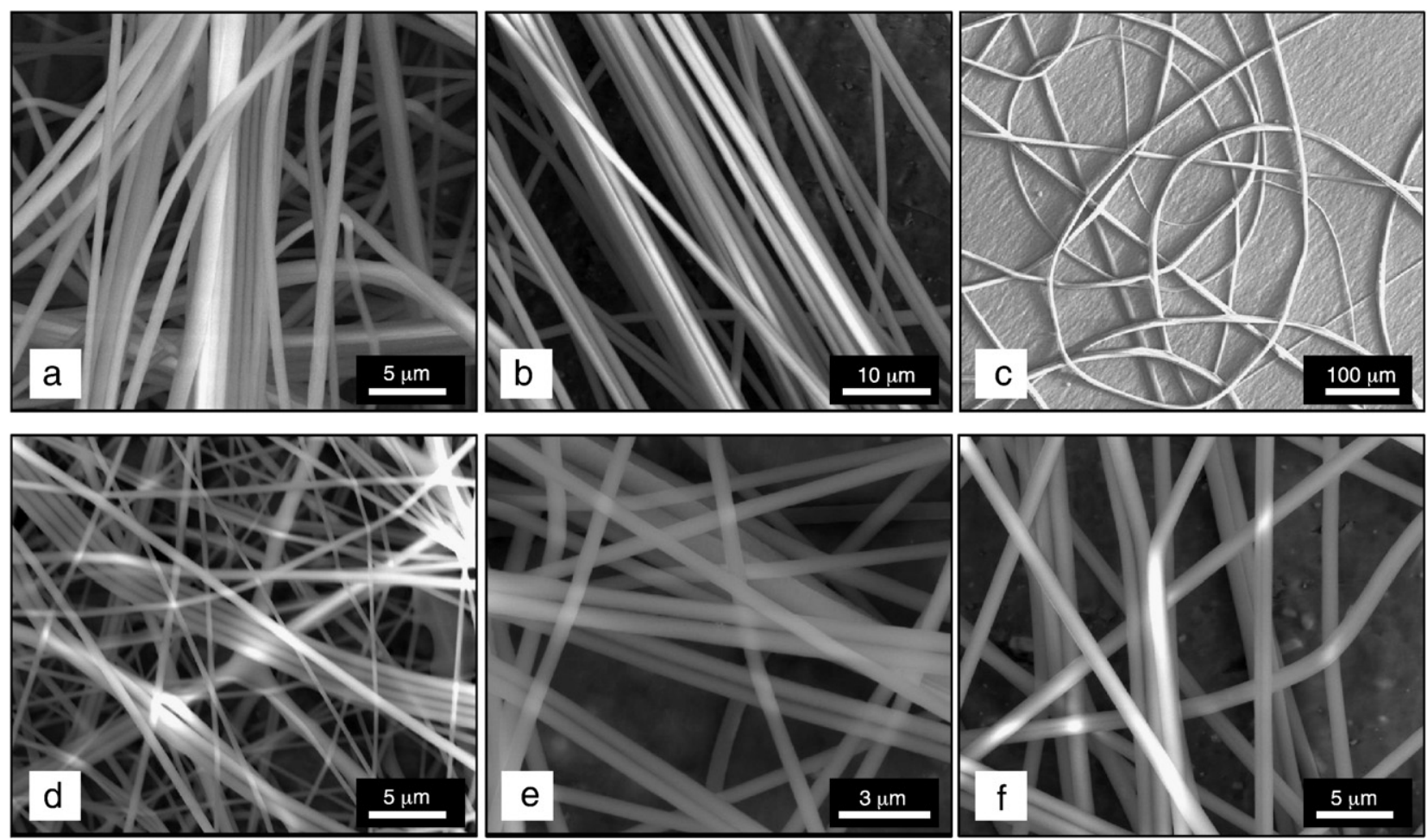

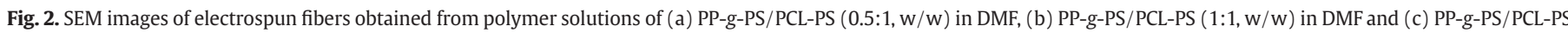

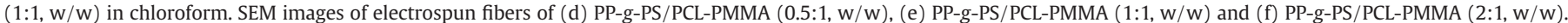


the results when PS is used as the matrix (Fig. 2a-b). We suggest that the overall charge density of the fibers in this case is higher due to the polar nature of PMMA that may induce a more intense dipole-dipole interaction with the polar DMF solvent as compared to the case for PS. This may induce a slower rate of evaporation and a higher amount of the residual solvent of the deposited fibers. The presence of the PMMA in the blend increases the dielectric constant (3.9 compared to 2.56 for PS). The larger dielectric constant leads to a larger Coulombic repulsion force, i.e. a higher electrostatic repulsion, that overcomes the molecular cohesion resulting in a decreased tendency for bundling of the fibers.

\section{Conclusion}

In summary, self-aligned bundled fibers of PP-g-PS/PCL-PS and PP$g$-PS/PCL-PMMA were obtained by the electrospinning technique. The self-alignment and bundling characteristics of these electrospun fibers were attributed to the unique molecular architecture of the conjugated polymer; PP-g-PS/PCL, and its peculiar interactions with the solvent (DMF) and the polymer matrixes (PS and PMMA) used for the electrospinning. The differences in fibers morphology for the two studied matrixes may reflect differences in self-assembly kinetics for the apolar and polar nature of PS and PMMA, respectively. Despite these differences, bundling and alignment at the macroscopic level was observed in both cases (being more effective in the case of PP-gPS/PCL-PS blend), demonstrating its dependence on the peculiar architecture and behavior of the used conjugated polymers in the studied blends. Future surface analyses will provide us further insightful information for understanding the bundling and sponta- neous alignment of these fibers. The optical properties of these fibers are also in our interest for the future studies.

\section{Acknowledgments}

We gratefully acknowledge The Danish Advanced Technology Foundation for funding the project NanoNonwovens, collaboration with Fibertex A/S, and the Danish Research Agency for the funding to the iNANO center.

\section{References}

[1] Greiner A, Wendorff JH. Angew Chem Int Ed 2007;46:5670-703.

[2] Li D, Xia YN. Adv Mater 2004;16:1151-70.

[3] Ramakrishna S, Fujihara K, Teo WE, Lim TC, Ma Z. An Introduction to Electrospinning and Nanofibers. World Scientific Publishing Company; 2005.

[4] Xin Y, Huang Z, Chen J, Wang C, Tong Y, Liu S. Mater Lett 2008;62:991-3.

[5] Chronakis IS, Grapenson S, Jakob A. Polymer 2006;47:1597-603.

[6] Babel A, Li D, Xia YN, Jenekhe SA. Macromolecules 2005;38:4705-11.

[7] Gonzales R, Pinto NJ. Synth Met 2005;151:275-8.

[8] Jang SY, Seshadri V, Khil MS, Kumar A, Marquez M, Mather PT, et al. Adv Mater 2005;17:2177-80.

[9] Demirel AL, Yurteri S, Cianga I, Yagci Y. J Polym Sci Part A Polym Chem 2007;45: 2091-104.

[10] Yurteri S, Cianga A, Demirel AL, Yagci Y. J Polym Sci Part A Polym Chem 2005;43: 879-96.

[11] Reneker DH, Chun I. Nanotechnology 1996;7:216-23.

[12] Li D, Wang YL, Xia YN. Nano Lett 2003;3:1167-71.

[13] Xin Y, Huang ZH, Li WW, Jiang ZJ, Tong YB, Wang C. Eur Polym J 2008;44:1040-5.

[14] Ruotsalainen T, Turku J, Heikkila P, Ruokolainen J, Nykanen A, Laitinen T, et al. Adv Mater 2005; 17:1048-52.

[15] Jarusuwannapoom T, Hongroijanawiwat W, Jitjaicham S, Wannatong L, Nithitanakul M, Pattamaprom C, et al. Eur Polym J 2005;41:409-21. 